\title{
FOLIA
}

Amazónica

Instituto de Investigaciones de la Amazonía Peruana

Vol. 27 (1) 2018. 1-8

DOI: https://doi.org/10.24841/fa.v27i1.438

\section{EL PH Y LA ABSORCIÓN DE CADMIO EN ALMENDRAS DE CACAO ORGÁNICO (Theobroma cacao L.) EN LEONCIO PRADO, HUÁNUCO, PERU}

\author{
Nelino FLORIDA ROFNER ${ }^{1}$, Shilthon Lineker CLAUDIO MELCHOR ${ }^{1}$, \\ Raúl GÓMEZ BERNAL ${ }^{2}$ \\ 1 Universidad Nacional Agraria de la Selva. Av. Universitaria km 1,5 carretera Tingo María, Huánuco. \\ E-mail: nelinof@hotmail.com \\ 2 Asociación de Productores Cacao Alto Huallaga. Área de Proyectos.
}

\section{RESUMEN}

El tipo de suelo tiene importante influencia en la disponibilidad y absorción del cadmio por la planta de cacao (Theobroma cacao L.), en particular el pH, el \% de arcilla y la cantidad de $\mathrm{Mg}^{2+}$ y $\mathrm{Zn}$ presente en el suelo. Por ello, el objetivo fue medir la influencia del pH del suelo en los niveles de absorción del cadmio en granos de cacao CCN-51 en la provincia de Leoncio Prado, Huánuco (Perú). Para ello se seleccionaron 20 parcelas agrícolas de los socios de la Cooperativa Cacao Alto Huallaga, que cumplían con todas las normas y técnicas de producción orgánica. Se analizaron 20 muestras de suelos y de almendras, los resultados muestran que el pH promedio del suelo fue de 5.68; el cadmio disponible en el suelo fue de $0.32 \mu \mathrm{g} \mathrm{g}^{-1} \mathrm{y}$ el contenido de cadmio en las almendras de cacao de las 20 muestras analizadas arrojó $0.98 \mu \mathrm{g} \mathrm{g}^{-1}$. La investigación concluye que los valores de cadmio en almendras se encuentran encima del nivel máximo permitido por la unión europea y se encontró correlación negativa entre el pH del suelo y el contenido de cadmio en almendras de cacao $\left(\mathrm{R}^{2}=0.0522\right)$, no encontrándose una dependencia del cadmio en almendra, con respecto al $\mathrm{pH}$ del suelo, esto requiere seguir investigando para determinar los factores que intervienen en este proceso.

PALABRAS CLAVES: pH del suelo, cadmio en almendras, cacao CCN-51. 


\title{
THE pH AND ABSORPTION OF CADMIUM IN ALMONDS OF ORGANIC COCOA (Theobroma cacao L.), IN LEONCIO PRADO, HUÁNUCO, PERÚ
}

\begin{abstract}
The type of soil has an important influence on the availability and absorption of cadmium by the cacao plant (Theobroma cacao L.), in particular the $\mathrm{pH}$, the \% of clay and the amount of $\mathrm{Mg}^{2+}$ and $\mathrm{Zn}$ present in the soil. Therefore, the objective was to measure the influence of soil pH on the absorption levels of cadmium in CCN-51 cocoa beans in the Leoncio Prado - Huánuco province (Perú). For which 20 agricultural parcels were selected from the members of Cooperativa Cacao Alto Huallaga, which complied with all the norms and techniques of organic production. 20 soil samples and 20 samples of almonds were analyzed. The results show that the average $\mathrm{pH}$ of the soil was 5.68; the cadmium available in the soil was $0.32 \mu \mathrm{g} \mathrm{g}^{-1}$ and the cadmium content in the cocoa almonds of the 20 samples analyzed yielded $0.98 \mu \mathrm{g} \mathrm{g}^{-1}$. The investigation concludes that the values of cadmium in almonds are above the maximum level allowed by the European Union and a negative correlation was found between the $\mathrm{pH}$ of the soil and the content of cadmium in cocoa almonds ( $R 2=0.0522)$, not finding a dependence of cadmium in almonds, with respect to the $\mathrm{pH}$ of the soil, this requires further research to determine the factors involved in this process.
\end{abstract}

KEYWORDS: Soil pH, Cadmium in almonds, Cocoa CCN-51. 


\section{INTRODUCCIÓN}

El cacao (Theobroma Cacao.) es uno de los cultivos de gran importancia económica para el Perú, el análisis realizado por el MINAGRI (2016) destaca que la producción nacional de cacao entre los años 2009 y 2015, presentó un aumento de 36,8 mil toneladas a 87,3 mil toneladas en el 2015, alcanzando una extensión de 121,3 mil hectáreas con un rendimiento medio a $720 \mathrm{~kg} / \mathrm{ha}$. Destacando básicamente las regiones de San Martín con el 43\%; Junín con el 18\%, Cusco con $9 \%$, Ucayali con $8 \%$ y Huánuco con $6 \%$. Estas cinco regiones representan el $84 \%$ de la producción nacional y se exportó un volumen récord de 56,5 mil toneladas, incrementándose en este periodo un $62 \%$ por año. Este comportamiento continúa, pues MINAGRI (2017) señala que la producción nacional alcanzo 108, 677 toneladas exportándose 57, 324 toneladas.

Sin embargo, la Unión Europea aprobó la nueva reglamentación sobre límites máximos de cadmio en cacao y chocolate, Reglamento UE de la Comisión No. 488 / 2014 que modifica el Reglamento CE No. 1881/2006 y fija que a partir del 1 de enero del 2019 entrará en rigor para la UE, la norma considera como contenido máximo de cadmio en diversos productos de cacao en un rango de 0.10 a $0.80 \mu \mathrm{g} \mathrm{g}^{-1}$ (Jiménez, 2015). Por lo tanto, esto se convierte en una preocupación nacional, toda vez que los principales mercados de destino de las exportaciones de cacao en grano del Perú, destaca la Unión Europea (UE) que concentra el $76 \%$ de las exportaciones totales (MINAGRI, 2016). Por lo tanto, es necesario investigar para generar conocimientos que permitan comprender la dinámica de este elemento e intentar disminuir los niveles de $\mathrm{Cd}^{2+}$ en almendras de cacao.

En este contexto, la principal forma química del cadmio en la solución del suelo es $\mathrm{Cd}^{2+}$ y en condiciones naturales existe en promedios de
$<1 \mu \mathrm{g} \mathrm{g}^{-1}$, generalmente asociado al Zinc (Madeddu, 2005). Sin embargo, la mayoría de investigadores, como Huamani et al. (2012) señalan que el tipo de suelo tiene importante influencia en la disponibilidad y absorción por la planta. Además, Arévalo et al. (2016) señala que entre los parámetros del suelo que presentan correlación con la concentración de metales pesados, como el cadmio, es el pH, el \% de arcilla y la cantidad de $\mathrm{Mg}^{2+}$ presente. Por lo tanto, el trabajo tiene como objetivo, evaluar la influencia del pH del suelo en los niveles de absorción del cadmio en granos de cacao CCNN-51 (Theobroma cacao.) orgánico, en la provincia Leoncio Prado, Huánuco.

\section{MATERIALES Y MÉTODOS}

\section{Área de estudio}

La investigación se desarrolló en el último trimestre del 2017, en la provincia de Leoncio Prado, ubicada en el departamento de Huánuco, Las condiciones climáticas en la que se desarrolló este proyecto son: precipitación anual promedio de $3,428.8 \mathrm{~mm}$, temperatura media anual de $24,5^{\circ} \mathrm{C}$ y una humedad relativa promedio de $77 \%$, según la estación meteorológica José Abelardo Quiñones (Universidad Nacional agraria de la Selva). Presenta régimen bimodal, las mayores precipitaciones se producen entre los meses de septiembre a abril y la temporada seca abarca desde los meses mayo a agosto.

Las características de las parcelas evaluadas, fisiográficamente se encuentran en terrazas bajas y medias de origen aluvial y lomadas de origen coluvial; taxonómicamente el ámbito de la investigación corresponde a suelos Entisoles e Inceptisoles (GOREHCO 2016). Las plantaciones de cacao corresponden al clon CCN-51 con un aproximado de 7 años de producción, con certificación orgánica y con una producción promedio de $500 \mathrm{~kg} / \mathrm{ha}$. 
Tabla 1. Propietarios y lugar de extracción de las muestras de suelo y almendras

\begin{tabular}{|c|c|c|c|}
\hline Muestra (Código) & Productor & Ubicación & Clon \\
\hline PITEIS 01 & Clorinda Jesús Ruiz & Pueblo Nuevo & $\mathrm{CCN}-51$ \\
\hline PITEIS 02 & Cinthia Kiani Isidro Ushiñagua & Castillo Grande & $\mathrm{CCN}-51$ \\
\hline PITEIS 03 & Leoncio Condezo Luciano & Luyando & $\mathrm{CCN}-51$ \\
\hline PITEIS 04 & Carlos Gómez Bernal & Rupa Rupa & $\mathrm{CCN}-51$ \\
\hline PITEIS 05 & Margot Álvarez Salas & Castillo Grande & $\mathrm{CCN}-51$ \\
\hline PITEIS 06 & Mendis Paredes Arce & Castillo Grande & $\mathrm{CCN}-51$ \\
\hline PITEIS 07 & Augusto Ascencio Bedoya & Pueblo Nuevo & $\mathrm{CCN}-51$ \\
\hline PITEIS 08 & Gabriel Borrovich Rivera & Luyando & $\mathrm{CCN}-51$ \\
\hline PITEIS 09 & Maximiliano Gamarra Trujillo & Luyando & $\mathrm{CCN}-51$ \\
\hline PITEIS 10 & Daría Solís Hermosilla & Pueblo Nuevo & $\mathrm{CCN}-51$ \\
\hline PITEIS 11 & Juan Luis Ramírez Morales & José Crespo y Castillo & $\mathrm{CCN}-51$ \\
\hline PITEIS 12 & Jorge David Salazar Lino & Luyando & $\mathrm{CCN}-51$ \\
\hline PITEIS 13 & José Natividad Pérez & Luyando & $\mathrm{CCN}-51$ \\
\hline PITEIS 14 & Arnulfo Gamarra Trujillo & Luyando & $\mathrm{CCN}-51$ \\
\hline PITEIS 15 & María Barrueta Cajas & Pueblo Nuevo & $\mathrm{CCN}-51$ \\
\hline PITEIS 16 & Lucía Espinoza Sabino & Mariano Dámaso Beraún & $\mathrm{CCN}-51$ \\
\hline PITEIS 17 & Hernán Serrano Domínguez & Nuevo Progreso & $\mathrm{CCN}-51$ \\
\hline PITEIS 18 & Fernando Tenazoa Jesús & Irazola & $\mathrm{CCN}-51$ \\
\hline PITEIS 19 & Segundo Pinedo Ortega & Nuevo progreso & $\mathrm{CCN}-51$ \\
\hline PITEIS 20 & Rubén Delgado Paredez & Irazola & $\mathrm{CCN}-51$ \\
\hline
\end{tabular}

\section{Muestreo de suelos}

El muestreo de suelos siguió los criterios de la metodología propuesta por el USDA, extrayendo 20 submuestras por hectárea, a una profundidad de $0-20 \mathrm{~cm}$ y para evaluar el $\mathrm{pH}$ y $\mathrm{Cd}$ en el laboratorio de Suelos de la UNAS.

Para el análisis de cadmio, se utilizó la metodología ISO 11466-Soil Quality. Al extracto resultante se realizó la lectura en Espectrofotómetro de Absorción Atómica. Para el análisis de $\mathrm{pH}$ del suelo se aplicó el método del potenciómetro con una relación suelo/agua de 1:1. Finalmente, para el análisis de cadmio en granos de cacao se aplicó la digestión húmeda con solución ácida nítrico perclórico, siguiendo la metodología de MITE (2010). El extracto, al igual que en la muestra de suelo han sido leídos en el equipo de espectrofotometría de Absorción Atómica (SAYVET, 2013).

\section{Análisis estadístico}

Se utilizó un diseño completamente aleatorizado DCA en donde el tratamiento lo constituye las parcelas de cacao CCN-51, con tamaño de muestra $n=20$ (muestras de suelo y almendra). Los datos de niveles de $\mathrm{pH}$ y los contenidos de $\mathrm{Cd}^{2+}$ del suelo y planta fueron sometidos a análisis de correlación de Pearson $(p<0.05)$. 


\section{RESULTADOS Y DISCUSIÓN}

En la Tabla 2, se muestra el valor medio de $\mathrm{pH}=5.68$, que corresponde a un nivel moderadamente acido (SAGARPA, 2012), valores típicos de la parte tropical de la zona central del Perú. Al respecto, el IIAP (1995) señala que, en suelos de terraza alta y zonas de pendiente del Alto Huallaga, encontramos inceptisoles ácidos bien drenados como los Dystropepts. Estos valores también, son corroborados por Arévalo et al. (2016) al evaluar los suelos de las principales áreas de producción de cacao del Perú, en ella reporta que los valores menores $(\mathrm{pH}<7)$ se encontró en Piura (Huancabamba), Amazonas,

Tabla 2. Niveles de $\mathrm{pH}$ y contenido de cadmio disponible en el suelo

\begin{tabular}{|c|c|c|}
\hline Muestra (Código) & $\mathbf{p H ~ ( 1 : 1 )}$ & $\mathbf{C d}^{\mathbf{2 +}}\left(\boldsymbol{\mu} \mathbf{~ g}^{\mathbf{1}}\right)$ \\
\hline PITEIS 01 & 7.64 & 0.41 \\
\hline PITEIS 02 & 7.53 & 0.29 \\
\hline PITEIS 03 & 7.37 & 0.37 \\
\hline PITEIS 04 & 7.31 & 0.64 \\
\hline PITEIS 05 & 5.73 & 0.17 \\
\hline PITEIS 06 & 5.41 & 0.82 \\
\hline PITEIS 07 & 6.82 & 0.16 \\
\hline PITEIS 08 & 7.44 & 0.15 \\
\hline PITEIS 09 & 5.72 & 0.10 \\
\hline PITEIS 10 & 4.75 & 0.21 \\
\hline PITEIS 11 & 4.09 & 0.02 \\
\hline PITEIS 12 & 4.88 & 0.89 \\
\hline PITEIS 13 & 4.06 & 0.91 \\
\hline PITEIS 14 & 4.44 & 0.07 \\
\hline PITEIS 15 & 4.24 & 0.06 \\
\hline PITEIS 16 & 5.33 & 0.40 \\
\hline PITEIS 17 & 5.95 & 0.23 \\
\hline PITEIS 18 & 5.76 & 0.18 \\
\hline PITEIS 19 & 4.28 & 0.12 \\
\hline PITEIS 20 & 4.76 & 0.13 \\
\hline MEDIA & $\mathbf{5 . 6 8}$ & $\mathbf{0 . 3 2}$ \\
\hline SEM & $\mathbf{\pm 0 . 2 8}$ & $\mathbf{\pm 0 . 0 6 3}$ \\
\hline
\end{tabular}

SEM = Error estándar de la media
San Martín (El Dorado y Tocache), Huánuco, Junín y Cuzco. También, Huamani et al. (2012) evaluó suelo bajo producción de cacao entre Huánuco y San Martín encontrando una media de $\mathrm{pH}=6.05$, considerado como moderadamente acido (SAGARPA, 2012). Por lo tanto, el valor medio encontrado representa valores típicos de las áreas destinadas a la producción de cacao en la provincia de Leoncio Prado.

Con referencia del cadmio en suelos, la Agencia del Medio Ambiente de los Estados Unidos de Norteamérica (USEPA) estableció como nivel crítico en $0.43 \mu \mathrm{g}$. $\mathrm{g}^{-1}$ de cadmio total en suelos agrícolas (USEPA, 2002). También, la Unión Europea UE, a través de la directiva de Kelley, indica que los valores típicos en suelos no contaminados de cadmio están entre 0 y $1 \mu \mathrm{g}$. $\mathrm{g}^{-1}$ (Acevedo et al., 2005). Por lo tanto, los valores encontrados de $\mathrm{Cd}^{2+}$ en esta investigación presenta una media de $0.32 \mu$ g. $^{-1}$ (Tabla 2), considerándose como niveles bajos o suelos no contaminados por $\mathrm{Cd}^{2+}$. Sin embargo, al hacer la comparación con otras investigaciones, es inferior a lo reportado en esta misma zona por Huamaní et al. (2012), valores de cadmio promedio de $0.53 \mu \mathrm{g}$. $\mathrm{g}^{-1} \mathrm{y}$ considera a sus resultados como un contenido bajo. Igualmente, Crozier et al. (2012), quienes encontraron valores más altos de $\mathrm{Cd}^{2+}$ en la Zona Norte $(0.79 \mu \mathrm{g}$. g - 1$)$ y menores en la Zona Central $(0.68 \mu \mathrm{g}$. g -1) y

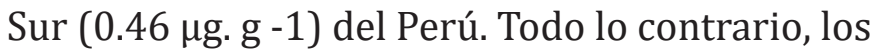
resultados de Arévalo et al. (2016), reporta que el cadmio ha sido el metal menos frecuente en los suelos muestreados. Valores nulos de $0.00 \mu \mathrm{g}$. $\mathrm{g}^{-1}$ encontró en Cajamarca (San Ignacio), San Martín (El Dorado y Tocache), Huánuco y Cuzco.

El análisis de correlación (Tabla 3), muestra que entre el $\mathrm{pH} \mathrm{y} \mathrm{Cd}^{2+}$ en suelo no existe correlación significativa. Este comportamiento es contrario a lo reportado por Arévalo et al. (2016), pues en sus resultados se observaron diferencias estadísticamente significativas $(\mathrm{p}<0.05)$ entre el cadmio del suelo y el $\mathrm{pH}$. Al respecto, si bien 
Tabla 3. Análisis de correlaciones entre el pH y contenido de cadmio disponible en suelo y almendras de cacao

\begin{tabular}{|c|c|c|c|c|}
\hline & & $\mathrm{pH}$ & $\begin{array}{l}\mathrm{Cd}^{2+} \text { en } \\
\text { suelo }\end{array}$ & $\begin{array}{l}\mathrm{Cd}^{2+} \text { en } \\
\text { almendra }\end{array}$ \\
\hline & Correlación de Pearson & 1 & ,050 &,- 228 \\
\hline $\mathrm{pH}$ & Sig. (bilateral) & & ,835 & ,333 \\
\hline & N & 20 & 20 & 20 \\
\hline
\end{tabular}

Otras investigaciones concuerdan con nuestro resultado, como el de Tantalean \& Huauya (2017) quienes reportan contenido de cadmio en almendras de parcelas ubicadas en suelo residual de 0,84 $\mu \mathrm{g}$. $\mathrm{g}^{-1}$, y en el suelo aluvial de 1,08 $\mu$ g. $\mathrm{g}^{-1}$ (para áreas ubicadas entre Huánuco y San Martin). Valores aún más altos reporta Lanza et al. (2016), concentraciones de $\mathrm{Cd}^{2+}$ ubicadas en un rango entre 0,95 y 2,09 $\mu \mathrm{g}$. $\mathrm{g}^{-1}$. sin embargo, estos

el $\mathrm{pH}$ encontrado no se correlaciona con $\mathrm{Cd}^{2+}$, puede explicarse teniendo en cuenta que la disponibilidad del $\mathrm{Cd}^{2+}$ en el suelo pueden estar influenciada por otros parámetros, como la presencia de materia orgánica y arcilla (Arévalo et al., 2016; Huamani et al., 2012; Degryse et al., 2009). Además, el resultado encontrado concuerda con las directivas de Kelley, pues, para suelos con rango de $\mathrm{pH} 5-6$, considera que presenta ligera contaminación por $\mathrm{Cd}^{2+}$ (Acevedo et al., 2005), en este sentido los valores encontrados coinciden con esta directiva, pues tenemos niveles bajos de $\mathrm{Cd}^{2+}\left(0.32 \mu \mathrm{g} . \mathrm{g}^{-1}\right)$ y pH medio de 5.68, que está dentro del rango mencionado.

En la Tabla 4, se muestra el contenido de cadmio de muestras de almendra de cacao; cuyo contenido medio es de $0.98 \mu \mathrm{g}$. $\mathrm{g}^{-1}$; este nivel supera el límite máximo permisible de la Unión Europea (Reglamento Nº 488 / 2014) que establece como contenido máximo de cadmio en diversos productos de cacao en un rango de 0.10 a $0.80 \mu \mathrm{g}$. $\mathrm{g}^{-1}$ (Jiménez, 2015). Estos contenidos altos de cadmio en las almendras, para esta investigación no está relacionada con el pH (Tabla 3). Por lo tanto, atribuimos este nivel alto de $\mathrm{Cd}^{2+}$ fundamentalmente a otras características del suelo; como nivel de materia orgánica, CIC y la cantidad de arcilla presente (Arévalo et al., 2012; Huamani et al., 2012). resultados son valores altos en comparación a lo reportado por Zambrano (2017), en la localidad

Tabla 4. Niveles de $\mathrm{pH}$ del suelo y cadmio en almendras de cacao

\begin{tabular}{|c|c|c|}
\hline Muestra (Código) & $\mathrm{pH}(1: 1)$ & $\begin{array}{c}\mathrm{Cd}^{2+} \text { en almendra } \\
\left(\mu \mathrm{g} \cdot \mathrm{g}^{-1}\right)\end{array}$ \\
\hline PITEIS 01 & 7.64 & 1,37 \\
\hline PITEIS 02 & 7.53 & 0,61 \\
\hline PITEIS 03 & 7.37 & 0,38 \\
\hline PITEIS 04 & 7.31 & 1,25 \\
\hline PITEIS 05 & 5.73 & 0,52 \\
\hline PITEIS 06 & 5.41 & 0,18 \\
\hline PITEIS 07 & 6.82 & 0,91 \\
\hline PITEIS 08 & 7.44 & 0,30 \\
\hline PITEIS 09 & 5.72 & 0,30 \\
\hline PITEIS 10 & 4.75 & 1,72 \\
\hline PITEIS 11 & 4.09 & 0,43 \\
\hline PITEIS 12 & 4.88 & 0,20 \\
\hline PITEIS 13 & 4.06 & 6,70 \\
\hline PITEIS 14 & 4.44 & 0,18 \\
\hline PITEIS 15 & 4.24 & 0,70 \\
\hline PITEIS 16 & 5.33 & 1,52 \\
\hline PITEIS 17 & 5.95 & 0,58 \\
\hline PITEIS 18 & 5.76 & 0,85 \\
\hline PITEIS 19 & 4.28 & 0,45 \\
\hline PITEIS 20 & 4.76 & 0,53 \\
\hline Media & 5.68 & 0.98 \\
\hline SEM & \pm 0.28 & \pm 0.32 \\
\hline
\end{tabular}

SEM = Error estándar de la media 
de luz de américa-Ecuador, sus resultados mostraron niveles máximos de $0,41 \mu \mathrm{g}$. $\mathrm{g}^{-1} \mathrm{y}$ niveles mínimos de $0,31 \mu \mathrm{g}$. $\mathrm{g}^{-1}$ en $\mathrm{CCN}-51$ con diferente método de secado. Igualmente, Sofia \& Pablo (2013), en Esmeraldas-Ecuador encontró una media de cadmio en almendra de cacao de $0.35 \mu \mathrm{g}$. $\mathrm{g}^{-1}$, valor inferior a nuestros resultados.

La directiva de Kelley, se indica que conforme el pH aumenta la contaminación disminuye y a la vez las plantas absorben menos estos elementos (Acevedo,2005).Sinembargo, nuestrosresultados no coinciden con esta directiva. Finalmente, Recientes estudios demuestran que los suelos y granos en áreas de cultivo de cacao presentar altos niveles de metales pesados (Tantalean y Huauya, 2017; Arevalo et al., 2016; Crozier et al., 2012; Huamaní et al., 2012) lo que podría llevar a problemas de consumo y exportación hacia la comunidad Europea, de este producto a partir del 2019. Por lo tanto, es necesario seguir investigando para generar conocimiento básico que permita encontrar tecnologías para reducir los niveles de $\mathrm{Cd}^{2+}$ en almendras de cacao.

\section{CONCLUSIONES}

- El pH medio del suelo en las parcelas de cacao, presenta un nivel ligeramente acido (5.68) $y$ el nivel medio de cadmio disponible en el suelo fue de $0.32 \mu \mathrm{g}$. $\mathrm{g}^{-1}$, considerado bajos para USEPA (2002) y también para la directiva Kelley de la Unión Europea.

- El contenido de cadmio en las almendras de cacao de las 20 muestras analizadas arrojó $0.98 \mu \mathrm{g}$. g-1, mayor que el nivel máximo permitido por la Unión Europea (Reglamento $\mathrm{N}^{\circ} .488$ / 2014).

- No hay correlación entre el pH y $\mathrm{Cd}^{2+}$ en los suelos evaluados, asimismo no hay correlación entre el pH del suelo y el contenido de cadmio en las almendras de cacao.

\section{BIBLIOGRAFIA CITADA}

Acevedo, E.; Carrasco, M.; León, O.; Silva, P.; Castillo, G.; Ahumada, I.; Borie, G.; González, S. 2005. Informe de criterios de calidad de suelo agrícola. Servicio Agrícola y Ganadero, Chile. 205 pp.

Alloway, B. J. 2013. Heavy metals in Soils: Trace Metals and Metallloids in Soils and their Bioavailability, 3 ed. Springer, 614 pp.

Arévalo, G. E.; Meyier, E.; Obando, C.; Zúñiga, C. L.; Cesar, O.; Arévalo, H. C. O.; Baligar, V.; Zhenli, He. 2016. Heavy metals in soils of cocoa plantations (theobroma cacao l.) in three regions of peru. Ecología Aplicada, 15(2): 81-89.

Crozier, J. 2012. Heavy metals in Cocoa. International Workshop on Possible EU regulations on cadmium in cocoaand chocolate products. Nature and Food Quality in the Netherlands (LNV), the European Cocoa Association (ECA) and CAOBISCO.

Degryse, F.; Smolders, E.; Parker D. R. 2009. Partitioning of metals ( $\mathrm{Cd}, \mathrm{Co}, \mathrm{Cu}, \mathrm{Ni}, \mathrm{Pb}, \mathrm{Zn}$ ) in soils: concepts, methodologies, prediction and applications. A review. European Journal of Soil Science, 60(4): 590-612.

Gobierno Regional de Huánuco-GOREHCO. 2016. Zonificación ecológica económica base para el ordenamiento territorial de la región Huánuco. [Internet]. Provincias: Huánuco, Marañón, Leoncio Prado, Pachitea y Puerto Inca 260pp. Humani, H. A.; Huauya, M. A.; Mansilla, L. G.; Florida, R. N.; Neira, G. M. 2012. Presencia de Metales pesados en el cultivo de cacao (Theobroma cacao L.) orgánico. Acta Agronómica, 61(4):339-344.

Instituto de Investigaciones de la Amazonía Peruana (IIAP). 1995. El recurso del suelo en la amazonia peruana, diagnóstico para su investigación (segunda aproximación) Fernando Rodríguez Achung. Documento tecnico n. 14. Iquitos, Perú. 56 pp. 
Jiménez, T. C. S. 2015. Estado legal mundial del cadmio en cacao (Theobroma cacao): fantasía o realidad. Producción + Limpia. 10(01): 89104.

Lanza, J. G.; Churión, P. C.; Liendo, N. J.; López, V. H. 2016. Evaluación del contenido de metales pesados en cacao (teobroma cacao l.) de santa bárbara del zulia, Venezuela. Saber, Universidad de Oriente, Venezuela. Vol. 28, n. ${ }^{\circ}$ 1: 106-115.

Madeddu, R. 2005. Estudio de la influencia del cadmio sobre el medioambiente y el organismo humano. Universidad de Granada. 4 pp.

MINAGRI. 2017. Estudio del cacao en el Perú y en el mundo. Un Análisis de la Producción y el Comercio al 2017. Dirección Estudios Económicos e Información Agraria-DEEIA: $60 \mathrm{pp}$

MINAGRI. 2016. Estudio del cacao en el Perú y en el mundo; Situación Actual y Perspectivas en el Mercado Nacional e Internacional al 2015. MINAGRI-DGPA-DEEIA, 86 pp.

MITE, F., CARRILLO, M. \& W. DURANGO. 2010. Avances del monitoreo de presencia de cadmio en almendras de cacao, suelos y aguas en Ecuador. Actas XII Congreso Ecuatoriano de la Ciencia del Suelo. Santo Domingo, 17-19 de Noviembre del 2010.

REGLAMENTO (UE) n. ${ }^{\circ}$ 488/2014. De la comisión de 12 de mayo de 2014, que modifica el Reglamento (CE) n. ${ }^{\circ} 1881 / 2006$ por lo que respecta al contenido máximo de cadmio en los productos alimenticios. Diario Oficial de la Unión Europea, pp 5. Disponible en: https:// www.boe.es/doue/2014/138/L0007500079.pdf

Sayvet, V. B. 2013. Guía metodológica para el muestreo y detección de cadmio en suelos, agua, fertilizantes, almendras de cacao y productos derivados, Lima, 76 pp.

Secretaría de Agricultura, Ganadería, Desarrollo Rural, Pesca y Alimentación (SAGARPA). 2012. Subíndice de Uso Sustentable del Suelo Metodología de Cálculo. Componente: Línea de Base del Programa de Sustentabilidad de los Recursos Naturales. 58 pp.

Sofia, A.; Pablo, P. 2013. Determinación de Cadmio en la Almendra de Cacao (Theobroma cacao) de cinco fincas ubicadas en vía San Domingo, Esmeralda [tesis] Pontifica Universidad del Ecuador, facultad de ciencias exactas y naturaleza, Ecuador. 2013: 81 pg. [Citado el 6 de junio 2018]. Disponible en: file://C:/Users/Usuario/ Downloads/43-1-51-1-10-20170614.pdf

Tantalean, P. E.; Huauya R.M. 2017. Distribución del contenido de cadmio en los diferentes órganos del cacao CCN-51 en suelo aluvial y residual en las localidades de Jacintillo y Ramal de Aspuzana. Rev. de investig. agroproducción sustentable 1(2): 69-78.

United States Environmental Protection Agency (USEPA). 2002. Supplemental guidance for developing soil screening levels for superfund sites Available from http://www.epa.gov/ superfund/health/conmedia/soil/ind ex.htm.

Zambrano, V. E. 2017. Evaluación del contenido de cadmio en dos variedades de cacao (theobroma cacao L.) considerando distintos métodos de secado en la localidad de luz de américa [tesis]. universidad nacional de las fuerzas armadasecuador. 2017: 61p. [Citado el 10 de junio 2018]. Disponible en: https://repositorio. espe.edu.ec/bitstream/21000/12962/1/TESPE-002802.pdf

\section{Recibido: 1 de mayo de 2018 Aceptado para publicación: 26 de julio de 2018}

